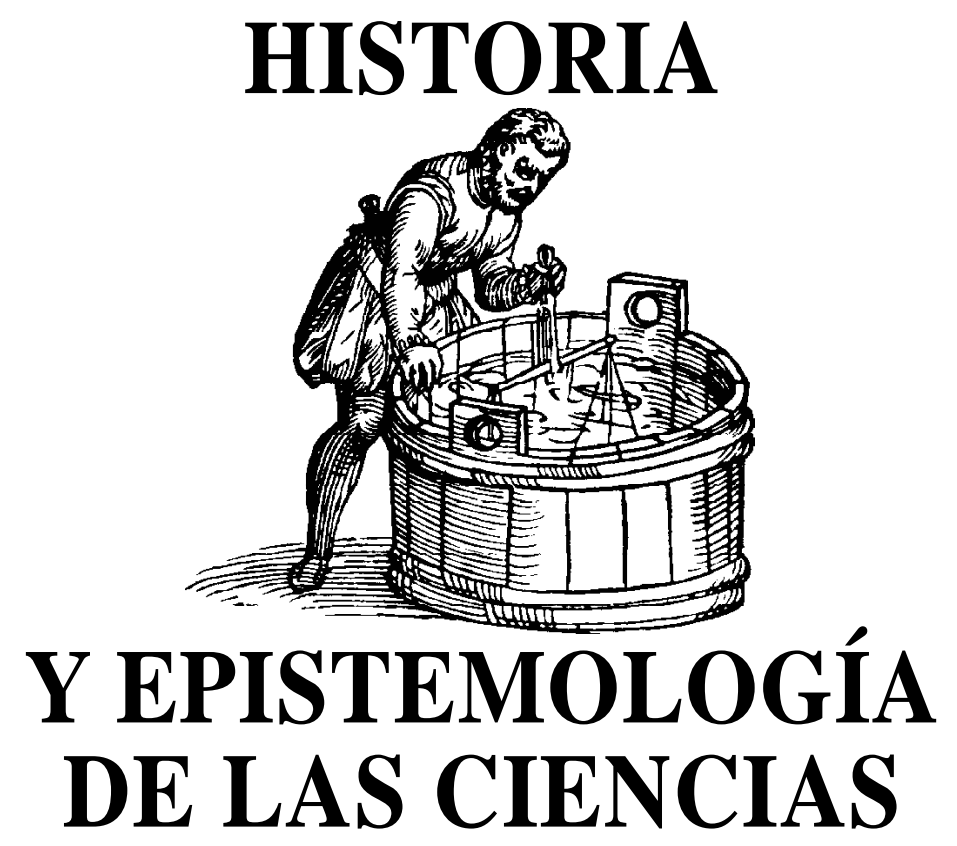

\title{
RESULTADOS OBTENIDOS INTRODUCIENDO HISTORIA DE LA CIENCIA EN LAS CLASES DE FÍSICA Y QUÍMICA: MEJORA DE LA IMAGEN DE LA CIENCIA Y DESARROLLO DE ACTITUDES POSITIVAS
}

SOLBES, J. Y TRAVER, M.

IES José Rodrigo Botet. 46940 Manises

IES S. Vicent Ferrer. Parc Salvador Castell, 16. 46680 Algemesí

E-mail: manelt@ctv.es

\section{SUMMARY}

This is a report about research and results in the application of History of Science to Science Teaching. After a first approach to analyse which is today's status of History of Science in high school Physics and Chemistry classes, we attempt to demonstrate that an appropriate introduction of several aspects of history and sociology of Science in our classes can operate a significant improvement in pupil's image and attitudes in Science and Science Teaching. We will show that several groups of pupils from 15 to 17 improve significantly their interest in Science after at least a year working with papers containing many different activities that involve different historical aspects of Science, i.e., context biographies, original papers, reports on STS in history or videos showing the making and growth of major concepts in Physics and Chemistry. 


\section{INTRODUCCIÓN}

El origen del tema se encuentra en una investigación empezada hace unos años (Solbes y Traver, 1996) en la cual se llevó a cabo el análisis crítico de la situación de la enseñanza habitual respecto a la falta de una perspectiva histórica adecuada que puso en evidencia la imagen deficiente de la naturaleza y evolución de la ciencia que se transmite a los alumnos y su influencia en el desinterés de los alumnos hacia el aprendizaje de la física y la química. Al mismo tiempo, estas conclusiones permiten vislumbrar cuáles podrían ser las soluciones que se debería aplicar para la mejora de las deficiencias detectadas.

No creemos que la ausencia de la historia de la ciencia en la formación inicial sea la razón preponderante de distorsión en la comprensión de la ciencia. Hay muchas otras razones: las finalidades de la enseñanza universitaria, sus contenidos (mayoritariamente conceptuales), el método usual de enseñanza, la forma de evaluación, sin olvidar la ausencia de la filosofía de la ciencia o de las relaciones CTS que sí se imparten en universidades de otros países. Lo que sí podemos afirmar es que el resultado de este largo proceso de «socialización» universitaria (que se inicia en BUP y COU) es, en muchas ocasiones, un licenciado científico bastante aislado de la realidad, centrado en el conocimiento de la naturaleza y que se preocupa poco profesionalmente de los problemas del mundo, como denunciaban recientemente algunos científicos en la prensa. Y cuando este licenciado se transforma en profesor «rechaza» como no científicas determinadas cuestiones, por urgentes que sean para el futuro de la humanidad. Y por eso han tenido y tienen tan poco éxito, entre el profesorado, los libros y proyectos que utilizan historia de la ciencia.

Dada la importancia de los materiales curriculares en la enseñanza-aprendizaje de las ciencias, nos planteamos como tema de trabajo la elaboración de materiales curriculares para la clase de física y química con la introducción de la historia de la ciencia. Si tenemos en cuenta que entre los objetivos de la LOGSE están los contenidos conceptuales, los procedimentales y los actitudinales, podemos constatar que la mayoría de propuestas curriculares existentes en el mercado siguen insistiendo únicamente en los contenidos conceptuales, y como mucho en los procedimentales, pero, en general, ignoran los contenidos actitudinales (Izquierdo, 1994). La propuesta de introducir aspectos de la historia de las ciencias puede ayudar a reforzar estos últimos. Pensamos que esto puede ser una pequeña contribución a uno de los mayores problemas que plantea la práctica educativa: la gran diversidad de los alumnos de la ESO, con alumnado desmotivado, con actitudes negativas e incluso con objetores escolares. Y, si no abordamos este problema, el profesorado (Romero, 1998) considerará la didáctica como academicista, al margen de la realidad educativa.

Esto nos ha llevado a un detenido estudio de la historia de la ciencia, pero sin olvidar que el objetivo de nuestro trabajo es una investigación en didáctica de las ciencias y no en historia de las ciencias. Por eso algunas afirma- ciones sobre los genios, el azar en la ciencia, etc. no pretenden establecer «verdades» históricas, sino aprovechar las aportaciones de la investigación en historia de las ciencias para mejorar la imagen y la enseñanza de la ciencia. Así, por ejemplo, respecto al problema de los genios, ya advierte la historia de la ciencia reciente contra el método de las grandes figuras (López Piñero y Navarro, 1995). No vamos a discutir sobre la relevancia de sus aportaciones a la ciencia, que es evidente, pero sí sobre la mitificación que se realiza de esas grandes figuras en los libros de texto, en los que se realizan reconstrucciones, más o menos racionales, de la ciencia a cargo de científicos. Algunos caminos de esa mitificación son atribuirles las contribuciones de científicos posteriores, como si las teorías ya naciesen completas en la mente de los «genios» (Truesdell, 1975). Lo preocupante de esto son sus posibles consecuencias didácticas: el alumnado puede tener la impresión de que la ciencia sólo es accesible a un reducido número de genios. Esto no parece la mejor forma de aproximar la ciencia a la mayoría de los ciudadanos, lo cual es un objetivo de la ESO. Por otra parte, la mayoría de las grandes figuras son varones (Spector, 1995), dado que la ciencia del pasado es la que ocupa el mayor tiempo de la enseñanza, y pertenecientes a otros países que no son el nuestro. Y esto también puede tener consecuencias en la enseñanza de las ciencias, por ejemplo: hacer que las ciencias físicas, ingenierías, etc., sean vistas como profesiones masculinas, provocando que asignaturas como la física y química de $4^{\circ}$ de ESO o la física de $2^{\circ}$ sean poco elegidos por las alumnas. Sobre el papel jugado por las mujeres científicas se puede encontrar información en Alic (1991), Schiebinger (1989) y Solsona (1997).

\section{PLANTEAMIENTO DEL PROBLEMA E HIPOTESIS DEL TRABAJO}

Los problemas que nos hemos planteado como continuación del trabajo antes citado son los siguientes: ¿Qué papel debe jugar la historia de las ciencias en la enseñanza de la física y química? ¿Qué consecuencias tiene la utilización de la historia de la ciencia en el alumnado, tanto en lo que se refiere a la imagen de la ciencia que tiene como en sus actitudes hacia ésta?

Según nuestra hipótesis creemos que es posible introducir aspectos de historia de la ciencia en la enseñanza de la física y la química para conseguir que los alumnos comprendan mejor la manera cómo se construye y se desarrolla la ciencia y qué repercusiones sociales tienen estos conocimientos. En consecuencia, pensamos que esto producirá una actitud positiva hacia los conocimientos científicos, que mejorará el ambiente del aula y el interés de los alumnos por participar en el proceso de enseñanza-aprendizaje, que lo hará más enriquecedor, de manera que integre las ciencias como parte inseparable del saber humano de carácter general. Del mismo modo, esperamos que este tratamiento recibirá una valoración positiva por parte del profesorado. 
La verificación de esta hipótesis consistirá, pues, en el diseño y aplicación a la enseñanza de materiales curriculares elaborados a partir de los criterios defendidos hasta aquí, de manera que incluyan diferentes actividades de historia de la ciencia. Estos materiales se han experimentado a lo largo de diferentes cursos en distintos institutos del País Valenciano con alumnos de los niveles de $2^{\circ}$ y $3^{\circ}$ de BUP y de COU para garantizar la aleatoriedad de la muestra. Para mejorar la validez estadística de los resultados de los grupos experimentales, además de los autores del trabajo, han intervenido otros 4 profesores y profesoras que empleaban los mismos materiales y metodología con diferentes grupos de alumnos.

Una vez utilizados los materiales a lo largo de un curso escolar, se trata de comparar los resultados obtenidos por estos grupos de alumnos experimentales y los resultados ya expuestos en la primera parte del trabajo (Solbes y Traver, 1996; Traver, 1996), que sirven de grupos de control y que, obviamente, han seguido una enseñanza sin la utilización de estos materiales.

En el conjunto de esta segunda parte de la investigación se han implicado 233 alumnos experimentales, además de los de control, y 83 profesores en activo que han participado en diferentes cursos de formación.

Exponemos a continuación las consecuencias operativas que se derivan de esta segunda hipótesis, que pueden ser contrastadas experimentalmente:

1) Es posible diseñar materiales curriculares con un enfoque histórico y usarlos en las clases de física y de química de nivel secundario de manera que contengan diversos aspectos que muestren una imagen de las ciencias físico-químicas más próxima a la realidad y más de acuerdo con su evolución histórica.

A fin de concretar los aspectos susceptibles de ser modificados por la utilización de los nuevos materiales aclararemos que, a fin de cuentas, el uso de la historia de la ciencia permitirá a los alumnos tener una nueva imagen de la ciencia y de los científicos más próxima a la realidad, lo cual implicará:

a) Considerar la ciencia como una construcción sistemática de conocimientos elaborada a lo largo de la historia y no como un conjunto de descubrimientos más o menos fortuitos de realidades preexistentes.

b) Reconocer los problemas significativos que hay en la base de la construcción de los conceptos más importantes y de las principales teorías científicas y que en diferentes momentos históricos han abierto las líneas de investigación más productivas.

c) Atribuir a los experimentos científicos un valor apropiado como una etapa más en el proceso de resolución de un problema, relativizar los resultados dentro de sus límites de validez y no asignarles de manera irreflexiva el papel de experiencias cruciales. d) Otorgar al formalismo matemático el valor instrumental útil y necesario para profundizar en la descripción, operativización y desarrollo de las hipótesis elaboradas previamente de forma cualitativa.

e) Reconocer la existencia de crisis importantes en la evolución histórica de los conocimientos científicos, tanto en el ámbito general de los principales paradigmas como en el interior mismo de éstos y relativizar el carácter acumulativo de los conocimientos científicos. Reconocer, en fin, que la evolución de los conocimientos científicos a lo largo de la historia no es un proceso lineal y que se han producido crisis importantes en los llamados paradigmas científicos, de igual modo que las ideas dentro de un mismo paradigma no siempre han sido inmutables y se han generado controversias que han producido modificaciones significativas.

f) Reconocer el carácter colectivo del trabajo de los científicos, donde es constante el intercambio de ideas, dada su provisionalidad, y el contraste de opiniones firmemente fundamentadas en trabajos de orígenes diversos cuyos resultados convergen y se muestran coherentes.

g) Reconocer algunas implicaciones sociales de las principales aportaciones del pensamiento científico al desarrollo general de la humanidad y la fuerza de éste para transformar nuestra percepción del mundo y, en definitiva, tener una visión más humanizada de la ciencia.

h) Valorar adecuadamente las contribuciones de los científicos de nuestro país que han aportado a la ciencia los frutos de su trabajo serio y con el rigor adecuado, a pesar de ser generalmente desconocidos, per razones diversas de carácter histórico.

i) Valorar adecuadamente las contribuciones de las mujeres científicas, habitualmente ignoradas por una visión de la ciencia con excesiva frecuencia centrada en determinadas épocas y en ciertos tópicos masculinos.

2) La utilización en les clases de física y química de los materiales elaborados producirá cambios en los alumnos experimentales. Como consecuencia de todo ello, es posible que las alumnas y los alumnos tengan una visión más acertada de cómo se construyen los conocimientos científicos, de sus repercusiones sociales y que mejoren su actitud hacia el estudio de la ciencia. Más concretamente los cambios que pensamos que se pueden producir en los alumnos serían:

a) Conocer mejor aspectos de historia de la ciencia, antes generalmente ignorados $\mathrm{y}$, consecuentemente, mostrar una imagen de la ciencia más completa y contextualizada.

b) Valorar adecuadamente aspectos internos del trabajo científico como: los problemas abordados, el papel del azar, la importancia de los experimentos, el formalismo matemático y la evolución de los conocimientos (crisis de paradigma, controversias y cambios internos). 
c) Valorar adecuadamente aspectos externos como: el carácter colectivo del trabajo científico, las implicaciones sociales de la ciencia, la actividad científica realizada en España y el papel de las mujeres científicas.

d) Presentar una imagen menos tópica de la ciencia y los científicos.

e) Mostrar más interés hacia el estudio de la física y la química.

f) Mejorar el clima del aula y la participación en el proceso de enseñanza-aprendizaje.

g) Valorar positivamente la utilización de aspectos de historia de la ciencia en les clases de física y química como forma de ayudar a aumentar su interés hacia el estudio de estas materias.

A continuación detallaremos los instrumentos que hemos empleado para contrastar estas consecuencias de la hipótesis.

\section{DISEÑO EXPERIMENTAL PARA CON- TRASTAR LA HIPÓTESIS}

Tal como hemos esquematizado en el apartado anterior, las líneas generales del diseño que aplicaremos para contrastar esta segunda hipótesis consisten en la elaboración de materiales curriculares para el uso en la clase de física y química de los niveles objeto de nuestra investigación y la contrastación de los cambios significativos operados en las muestras de alumnos experimentales, por medio de los cuestionarios ya utilizados con los alumnos en la primera parte del trabajo, que servirán de muestras de control. Para enriquecer la constatación de los cambios actitudinales y recoger el clima de trabajo de los alumnos, añadiremos algunos instrumentos de observación directa en el aula.

1. Elaboración y utilización de materiales que permiten la introducción de actividades de historia de la ciencia en las clases de física y química.

Los últimos años son diversos los grupos de trabajo e investigación de profesores que se han dedicado a elaborar diversas colecciones de actividades y propuestas de programas guía para la clase de física y química con una orientación constructivista y una metodología activa en la línea que hemos propuesto en la fundamentación de nuestra hipótesis. Estas colecciones han sido el punto de partida para buscar actividades con un contenido histórico, aunque la escasez de éstas nos ha llevado a elaborar nuevas actividades más adecuadas a los propósitos de nuestro trabajo concreto.

Las fuentes a las que hemos acudido a consultar algunas actividades son diversas publicaciones, como el proyecto Galaxia de Física y Química de ESO y Bachillerato (Calatayud et al., 1995, 1996, 1997 y 1998). También materiales del Grup Faraday (1988). Algunas activida- des también han sido elaboradas como parte del trabajo que lleva a cabo el Grupo Jeroni Munyós de física y química del CEP de Alzira - La Ribera.

En Traver (1996) presentamos una muestra variada de actividades con un contenido y orientación históricos que trata de cubrir los temas más importantes de los programas de BUP y COU y que, por tanto, pueden utilizarse en los diferentes niveles a lo largo de los temas correspondientes. (Presentamos algunas de ellas a título de ejemplo en el anexo). Muchas de estas actividades se han usado durante la investigación con los alumnos experimentales. Pero la inclusión de actividades de tipo histórico no se ha de limitar necesariamente a unas pocas actividades esporádicas, sino que es posible realizar un tema completo que utilice el hilo conductor histórico para la introducción adecuada de un determinado concepto científico.

Para disipar la preocupación detectada en muchos profesores respecto al posible retraso en el temario que implicaría aumentar los contenidos con aspectos históricos, aclararemos que no se trata de eso, sino más bien de reorientar los contenidos que se debe impartir empleando el hilo conductor histórico o como mínimo tenerlo presente en la introducción de determinados aspectos del tema que de igual modo se debe impartir, de manera que no hace falta incrementar los contenidos de los programas habituales, ya bastante sobrecargados. Así, entre los criterios que orientan la propuesta de un programa guía concreto incluimos los aspectos del proceso de construcción de los conocimientos y planteamos los problemas a investigar, las posibles ideas previas de los alumnos y la existencia de algún paralelismo con ideas vigentes en diferentes épocas, la introducción de conceptos y las dificultades y barreras epistemológicas que ha sido necesario vencer hasta llegar a establecerse, la resolución de problemas y los trabajos prácticos, pero también las posibles controversias que a lo largo de la historia se han generado, y utilizamos textos referidos a los autores o autoras de las diferentes contribuciones científicas, de manera que el mayor número de actividades posible esté impregnado de esta orientación histórica.

2. Diseño para contrastar si entre los alumnos de los grupos experimentales se produce una imagen más correcta de las ciencias físico-químicas y un aumento del interés hacia ellas.

En la primera parte de este trabajo (Solbes y Traver, 1996) quedó establecido el escaso papel que tiene la historia de la ciencia en la enseñanza habitual y cómo influye esto en la percepción que tienen los alumnos de la naturaleza de la ciencia. Por tanto, ahora trataremos de verificar que es posible contribuir a modificar la imagen de la ciencia que tienen los alumnos después de utilizar en las clases de física y química los nuevos materiales que tienen en cuenta estos aspectos mediante una metodología activa.

La verificación experimental de las consecuencias que se derivan de nuestra hipótesis se llevará a cabo por 
comparación de los resultados de los alumnos que han trabajado estos materiales en el aula (grupos experimentales) con los resultados de los alumnos que han seguido una enseñanza más tradicional, presentados en la primera parte del trabajo (grupos control) sin prácticamente enfoque alguno de tipo histórico.

La comprobación de los cambios producidos en los alumnos de los grupos experimentales se lleva a cabo con los cuestionarios A, B, C y D, ya utilizados en la primera parte del trabajo (Solbes y Traver, 1996), de los cuales mostraremos aquí sus objetivos principales. Los enunciados abreviados de los diferentes ítems se pueden leer en las tablas de resultados que acompañan este trabajo. Así el cuestionario A revelará si se han producido cambios significativos en la percepción de la naturaleza de la ciencia y el trabajo de los científicos por parte de los alumnos que han seguido un tratamiento con actividades y materiales de contenido histórico, ya que se han tenido en cuenta los diferentes ítems a la hora de elaborar los nuevos materiales.

Con el cuestionario B queremos verificar si la forma de crecimiento de la ciencia y los aspectos como las reper- cusiones sociales de los trabajos científicos y los problemas que los originaron han sido percibidos adecuadamente por los alumnos. Igualmente este cuestionario mostrará hasta qué punto han mejorado sus conocimientos sobre diferentes científicos y sus contribuciones a la ciencia.

El cuestionario $\mathrm{C}$ pondrá de manifiesto el grado de percepción que tienen los alumnos de aquellos aspectos más sociológicos de la evolución de la ciencia y las contribuciones de nuestro país.

Sin embargo, los cambios operados no serán únicamente de tipo conceptual y consideramos que también se habrán producido modificaciones de tipo actitudinal de manera que los alumnos mostrarán un mayor interés por las ciencias físico-químicas y su aprendizaje. Por eso el cuestionario D contribuirá a poner de manifiesto este cambio actitudinal. Para no verse influido por las respuestas del resto de cuestionarios, hemos propuesto este cuestionario en primer lugar y a continuación se ha pasado el resto de los cuestionarios en orden alfabético. Las condiciones en que se ha propuesto la resolución de los cuestionarios han sido las siguientes: a los alumnos

Tabla I

Análisis global del cuestionario de alumnos (A).

\begin{tabular}{|c|c|c|c|c|c|c|c|c|}
\hline \multirow{2}{*}{$\begin{array}{l}\text { PORCENTAJES } \\
\text { DE ALUMNOS } \\
\text { QUE: } \\
\begin{array}{l}\text { Creen que la ciencia } \\
\text { es descubrimiento (A1). }\end{array}\end{array}$} & \multicolumn{2}{|c|}{$\begin{array}{c}\text { GRUPO } \\
\text { CONTROL } \\
\% \begin{array}{c}(\mathbf{N}=479) \\
(\text { sd })\end{array}\end{array}$} & \multicolumn{2}{|c|}{$\begin{array}{c}\text { GRUPO } \\
\text { EXPER. } 1 \\
\%^{(\mathbf{N}=117)} \\
(\mathbf{s d})\end{array}$} & \multicolumn{2}{|c|}{$\begin{array}{c}\text { GRUPO } \\
\text { EXPER. } 2 \\
(\mathbf{N}=116) \\
\% \quad(\mathbf{s d})\end{array}$} & \multicolumn{2}{|c|}{$\begin{array}{r}\text { DIFERENCIAS } \\
\text { SIGNIFICATIVAS } \\
\alpha<\end{array}$} \\
\hline & 58,2 & $(2,3)$ & 37,6 & $(4,5)$ & 36,2 & $(4,5)$ & Sí & 0,001 \\
\hline $\begin{array}{l}\text { Creen que su objetivo } \\
\text { es formalista (A2). }\end{array}$ & 43,4 & $(2,3)$ & 26,5 & $(4,1)$ & 24,1 & $(4,0)$ & Sí & 0,001 \\
\hline $\begin{array}{l}\text { Creen que los trabajos } \\
\text { de Newton no se han } \\
\text { modificado (A3). }\end{array}$ & 42,4 & $(2,3)$ & 33,3 & $(4,4)$ & 45,7 & $(4,6)$ & $\begin{array}{l}\text { Exp. } 1 \\
\text { Exp. } 2\end{array}$ & $\begin{array}{l}0,10 \\
\text { No }\end{array}$ \\
\hline $\begin{array}{l}\text { Creen que el experimento } \\
\text { puede invalidar la teoría (A4). }\end{array}$ & 29,4 & $(2,1)$ & 15,4 & $(3,3)$ & 20,7 & $(3,8)$ & $\begin{array}{l}\text { Exp. } 1 \\
\text { Exp. } 2 \\
\end{array}$ & $\begin{array}{l}0,001 \\
0,05\end{array}$ \\
\hline $\begin{array}{l}\text { Creen que los conceptos } \\
\text { científicos no han sido } \\
\text { construidos (A5). }\end{array}$ & 49,5 & $(2,3)$ & 47,9 & $(4,6)$ & 39,7 & $(4,5)$ & $\begin{array}{l}\text { Exp.1 } \\
\text { Exp.2 }\end{array}$ & $\begin{array}{l}\text { No } \\
0,05\end{array}$ \\
\hline $\begin{array}{l}\text { Tienen una visión } \\
\text { acumulativa de la evolución } \\
\text { de la ciencia (A6). }\end{array}$ & 87,1 & $(1,5)$ & 5,1 & $(2,0)$ & 6,0 & $(2,2)$ & Sí & 0,001 \\
\hline
\end{tabular}

Fuente: Cuestionario de alumnos (A) para valorar la imagen implícita de la ciencia. 
Tabla II

Análisis global del cuestionario de alumnos (B).

\begin{tabular}{|c|c|c|c|c|c|c|}
\hline $\begin{array}{l}\text { PORCENTAJES } \\
\text { DE ALUMNOS } \\
\text { QUE: }\end{array}$ & \multicolumn{2}{|c|}{$\begin{array}{c}\text { GRUPO } \\
\text { CONTROL } \\
(\mathbf{N}=479)\end{array}$} & \multicolumn{2}{|c|}{$\begin{array}{l}\text { GRUPO } \\
\text { EXPERIMENTAL } \\
(\mathbf{N}=\mathbf{2 3 3})\end{array}$} & \multicolumn{2}{|c|}{$\begin{array}{c}\text { DIFERENCIAS } \\
\text { SIGNIFICATIVAS } \\
\alpha<\end{array}$} \\
\hline $\begin{array}{l}\text { Conocen alguna crisis } \\
\text { en la evolución de la ciencia (B1) }\end{array}$ & 15,7 & $(1,7)$ & 56,7 & $(3,2)$ & Sí & 0,001 \\
\hline $\begin{array}{l}\text { Citan cinco o más } \\
\text { científicos y sus trabajos (B2) }\end{array}$ & 28,8 & $(2,1)$ & 37,8 & $(3,2)$ & Sí & 0,02 \\
\hline $\begin{array}{l}\text { Citan correctamente todos } \\
\text { los autores propuestos (B3) }\end{array}$ & 4,4 & $(0,9)$ & 16,7 & $(2,4)$ & Sí & 0,001 \\
\hline $\begin{array}{l}\text { Conocen las repercusiones } \\
\text { sociales de algunos trabajos (B4) }\end{array}$ & 52,0 & $(2,3)$ & 71,6 & $(3,0)$ & Sí & 0,001 \\
\hline $\begin{array}{l}\text { Citan los problemas que originaron } \\
\text { algunos trabajos (B5) }\end{array}$ & 13,4 & $(1,6)$ & 40,8 & $(3,2)$ & Sí & 0,001 \\
\hline
\end{tabular}

Fuente: Cuestionario de alumnos (B) sobre desarrollo de la ciencia y contribuciones de los científicos.

experimentales se les ha propuesto la resolución conjunta de los cuatro cuestionarios, en una sesión, con el tiempo suficiente para contestar con tranquilidad; a los diferentes grupos, en un período del curso escolar semejante, hacia la mitad del último trimestre del curso escolar, una vez pasado el tiempo suficiente desde la realización de las diversas actividades propuestas en los materiales ensayados.

A fin de comprobar mejor los aspectos de la hipótesis referidos a las actitudes de los alumnos y el ambiente de trabajo y clima del aula, hemos decidido realizar la observación directa de una secuencia de clases en que se han realizado actividades de historia de la ciencia. El instrumento de observación diseñado consiste en una ficha de observación donde anotar o transcribir los diferentes aspectos que pueden ser significativos a la hora de contrastar el ambiente de la clase y el nivel de participación de los diferentes alumnos si comparamos una clase habitual con otra en que se han utilizado los materiales experimentales.

\section{RESULTADOS}

1) Como ya hemos mostrado mediante la bibliografía citada en el apartado anterior, es posible insertar actividades y transformar los temas que conformen el currículo de física y química con la elaboración de materiales que incorporen diversos aspectos históricos, como, por ejemplo, biografías contextualizadas que muestren los aspectos más humanos del trabajo científico, que integren textos originales de los autores, que muestren las controversias históricas y la evolución de determinados conceptos, etc., de manera que ayuden a la construcción de los conocimientos científicos y contribuyan a profundizar y reforzar estos conocimientos, teniendo en cuenta el contexto en que han surgido. Al utilizar estos materiales en el aula, hemos detectado los problemas de la falta de tiempo, no tanto por la introducción de la historia de la ciencia cuanto por la utilización de una metodología de investigación, activa, y el rechazo de algunos alumnos de COU o de $2^{\circ}$ de bachillerato a este tipo de actividades, señalando que están bien para cursos inferiores, pero que ellos deberían dedicar todo su tiempo a la preparación de las pruebas de acceso a la universidad.

2) Los alumnos que han seguido un curso de física y química con un tratamiento histórico, dentro del modelo didáctico basado en el cambio conceptual, metodológico y actitudinal muestran una imagen de la ciencia más contextualizada y próxima a la realidad y, en la mayoría de casos, se diferencian de forma significativa respecto a los alumnos que han seguido un curso que no contemplaba esta orientación. Los rasgos concretos que nos permiten hacer estas afirmaciones son:

a) Entre los alumnos que han seguido un curso en el cual se han incorporado actividades de tipo histórico (expe- 
Tabla III

Análisis global del cuestionario de alumnos (C).

\begin{tabular}{|c|c|c|c|c|c|c|c|c|}
\hline $\begin{array}{l}\text { PORCENTAJES } \\
\text { DE ALUMNOS } \\
\text { QUE: }\end{array}$ & \multicolumn{2}{|c|}{$\begin{array}{c}\text { GRUPO } \\
\text { CONTROL } \\
(\mathbf{N}=\mathbf{2 1 5})\end{array}$} & \multicolumn{2}{|c|}{$\begin{array}{l}\text { GRUPO } \\
\text { EXPER.1 } \\
(\mathbf{N}=117)\end{array}$} & \multicolumn{2}{|c|}{$\begin{array}{c}\text { GRUPO } \\
\text { EXPER. } 2 \\
(\mathbf{N}=116)\end{array}$} & \multicolumn{2}{|c|}{$\begin{array}{l}\text { DIFERENCIAS } \\
\text { SIGNIFICATIVAS }\end{array}$} \\
\hline $\begin{array}{l}\text { Conocen } 2 \text { o más modelos } \\
\text { con controversias ( } 1 \text { ) }\end{array}$ & 9,3 & $(2,0)$ & 17,9 & $(3,5)$ & 32,8 & $(4,4)$ & $\begin{array}{l}\text { Exp. } 1 \\
\text { Exp. } 2\end{array}$ & $\begin{array}{l}0,05 \\
0,001\end{array}$ \\
\hline $\begin{array}{l}\text { Conocen casos de apoyo } \\
\text { económico a la ciencia (C2) }\end{array}$ & 26,1 & $(3,0)$ & 40,2 & $(4,5)$ & 63,0 & $(4,5)$ & $\begin{array}{l}\text { Exp. } 1 \\
\text { Exp. } 2\end{array}$ & $\begin{array}{l}0,01 \\
0,001\end{array}$ \\
\hline $\begin{array}{l}\text { Argumentan a favor } \\
\text { del carácter colectivo (C3) }\end{array}$ & 35,8 & $(3,3)$ & 61,0 & $(4,5)$ & 71,6 & $(4,2)$ & Sí & 0,001 \\
\hline $\begin{array}{l}\text { Conocen científicos } \\
\text { españoles (C4) }\end{array}$ & 25,6 & $(3,0)$ & 28,2 & $(4,2)$ & 48,3 & $(4,6)$ & $\begin{array}{l}\text { Exp. } 1 \\
\text { Exp. } 2\end{array}$ & $\begin{array}{l}\text { No } \\
0,001\end{array}$ \\
\hline $\begin{array}{l}\text { Conocen relaciones } \\
\text { entre los avances técnicos } \\
\text { y los científicos (C5) }\end{array}$ & 47,9 & $(3,4)$ & 58,2 & $(4,6)$ & 68,1 & $(4,3)$ & $\begin{array}{l}\text { Exp. } 1 \\
\text { Exp. } 2\end{array}$ & $\begin{array}{l}0,10 \\
0,001\end{array}$ \\
\hline $\begin{array}{l}\text { Conocen facilidades } \\
\text { para la ciencia española (C6) }\end{array}$ & 4,7 & $(1,4)$ & 12,0 & $(3,0)$ & 14,7 & $(3,3)$ & $\begin{array}{l}\text { Exp. } 1 \\
\text { Exp. } 2\end{array}$ & $\begin{array}{l}0,05 \\
0,01\end{array}$ \\
\hline $\begin{array}{l}\text { Conocen impedimentos } \\
\text { a la ciencia española (C7) }\end{array}$ & 24,7 & $(2,9)$ & 29,9 & $(4,2)$ & 43,1 & $(4,6)$ & $\begin{array}{l}\text { Exp. } 1 \\
\text { Exp. } 2\end{array}$ & $\begin{array}{l}\text { No } \\
0,001\end{array}$ \\
\hline
\end{tabular}

Fuente: Cuestionario de alumnos (C) sobre aspectos de historia y sociología de la ciencia.

rimentales) se constata (Tabla I) una disminución significativa del porcentaje de los que perciben la actividad científica como descubrimiento (un $36,2 \%$ en uno de los grupos experimentales frente al 58,2 \% de los alumnos que no han seguido este curso [control]), reforzado por una disminución no tan destacada en el porcentaje de los que consideran que los conceptos científicos no se han creado sino que se han descubierto $(39,7 \%$ en uno de los grupos experimentales frente al $49,5 \%$ de los alumnos del grupo control). Igualmente el porcentaje de alumnos que atribuye un papel crucial a los experimentos ha disminuido hasta un 15,4\% de los alumnos experimentales, aunque el porcentaje de los alumnos de control que se mostraba de acuerdo con el falsacionismo ya era bajo $(29,4 \%)$.

b) La percepción del formalismo matemático como objetivo prioritario ha disminuido en los alumnos de los grupos experimentales y se sitúa entre el $24,1 \%$ y el $26,5 \%$, mientras que en los grupos control era del $43,3 \%$.

c) Los alumnos que después de haber seguido un curso con orientación histórica tienen aún una visión acumu- lativa de la evolución de la ciencia llegan hasta un $6 \%$, mientras que en los grupos control representan el $87,1 \%$ (Tabla II) y aumentan hasta el 56,7\%; los que recuerdan algunas crisis en la evolución de la ciencia y los que indican dos o más modelos con controversias alcanzan el $32,8 \%$ (Tabla III), mientras que en estos mismos aspectos los alumnos que no han seguido este curso presentaban unos porcentajes del $15,7 \%$ y del $9,3 \%$, respectivamente.

d) En los grupos experimentales el conocimiento de científicos y sus trabajos mejora (Tabla II), de manera que llegan a un $37,8 \%$ los que citan correctamente cinco o más científicos y al 16,7\% los que aciertan todos los autores de los trabajos propuestos, cuando en estos mismos aspectos los alumnos de control presentaban un $28,8 \%$ y un $4,4 \%$, respectivamente, de respuestas acertadas. El conocimiento de los trabajos de los científicos españoles (Tabla III) mejora de un 25,6\% de los alumnos de control a un 48,3\% de uno de los grupos experimentales. El carácter colectivo de la actividad científica es percibido correctamente por entre un $61 \%$ y un $71,6 \%$ de alumnos, según el grupo experimental, mientras que 
Tabla IV

Análisis global del cuestionario de actitudes (D).

\begin{tabular}{|c|c|c|c|c|c|c|}
\hline \multirow{2}{*}{$\begin{array}{l}\text { PORCENTAJES } \\
\text { DE ALUMNOS } \\
\text { QUE: } \\
\begin{array}{l}\text { Hacen una valoración muy } \\
\text { positiva de la enseñanza } \\
\text { recibida (D1) }\end{array}\end{array}$} & \multicolumn{2}{|c|}{$\begin{array}{c}\text { GRUPO } \\
\text { CONTROL } \\
(\mathbf{N}=215) \\
\% \quad \underset{(\text { sd })}{\%}\end{array}$} & \multicolumn{2}{|c|}{ 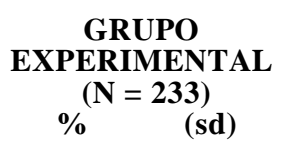 } & \multicolumn{2}{|c|}{$\begin{array}{c}\text { DIFERENCIAS } \\
\text { SIGNIFICATIVAS } \\
\alpha<\end{array}$} \\
\hline & 38,3 & $(3,3)$ & 64,4 & $(3,1)$ & SÍ & 0,001 \\
\hline $\begin{array}{l}\text { Creen que la historia de la ciencia } \\
\text { mejoraría su valoración (D2) }\end{array}$ & 61,3 & $(3,3)$ & 82,8 & $(2,5)$ & SÍ & 0,001 \\
\hline $\begin{array}{l}\text { Les gustaría conocer el proceso } \\
\text { de creación de la ciencia (D3a) }\end{array}$ & 17,0 & $(2,6)$ & 40,2 & $(3,2)$ & Sí & 0,001 \\
\hline $\begin{array}{l}\text { Les gustaría conocer } \\
\text { biografías de científicos (D3b) }\end{array}$ & 13,9 & $(2,4)$ & 24,7 & $(2,8)$ & SÍ & 0,01 \\
\hline $\begin{array}{l}\text { Les gustaría conocer aspectos } \\
\text { de CTS en la historia (D3c) }\end{array}$ & 5,3 & $(1,5)$ & 18,7 & $(2,6)$ & SÍ & 0,001 \\
\hline
\end{tabular}

Fuente: Cuestionario de alumnos (D) sobre el interés y actitudes hacia la ciencia.

entre los alumnos del grupo control era del 35,8\%. Por todo ello podemos considerar que se ha producido una mejora en la percepción de la visión más humanizada de la ciencia en aquellos alumnos que han seguido el curso con perspectiva histórica.

e) Entre estos alumnos también se puede detectar una mejor comprensión de los aspectos relacionados con la contextualización de los conocimientos científicos (Tabla II). Por ejemplo, un 58,6\% de alumnos puede señalar las repercusiones sociales de entre uno y tres casos propuestos y un $13 \%$ de cuatro o cinco casos, que en conjunto representan un 71,6\%, frente al $52 \%$ global que citaban los alumnos de los grupos control. También aumentan los alumnos que conocen los problemas generadores de diversos trabajos científicos (un 40,8\% cita al menos uno, frente al 13,4\% de los grupos control). Los casos de apoyo económico a la ciencia (Tabla III) son indicados por entre un $40,2 \%$ y un $63 \%$ de alumnos experimentales, mientras que, en los grupos control, el porcentaje era un $26,1 \%$, y las relaciones entre la ciencia y la técnica son conocidas por entre un $58,2 \%$ y un $68,1 \%$ de alumnos experimentales, frente al $47,9 \%$ de los grupos control. Las facilidades para la ciencia española son indicadas por un $14,7 \%$ de alumnos experimentales $(4,7 \%$ los de control) y los impedimentos los señala un $43,1 \%$ de uno de los grupos experimentales $(24,7 \%$ los de control).
3) En la tabla IV se observa que un elevado porcentaje de los alumnos y alumnas que han seguido el curso con orientación histórica (el 64,4\%) hace una valoración alta de la enseñanza recibida (frente al 38,3\% de los que no han seguido el curso) y la contribución de la perspectiva histórica a la mejora de la valoración la indica un $82,8 \%$, porcentaje que confirma y supera las buenas expectativas que tenían los alumnos no tratados $(61,3 \%)$. Los alumnos experimentales muestran un mayor interés por conocer aspectos como el proceso de creación de la ciencia $(40,2 \%)$, biografías de científicos $(24,7 \%)$ y relaciones CTS $(18,7 \%)$, mientras que los alumnos del grupo control que mostraban interés por estos mismos aspectos eran, respectivamente, el $17 \%$, el $13,9 \%$ y el $5,3 \%$. En conjunto, el porcentaje de respuestas que no manifestaban ningún interés por la historia se ha reducido del 44,9\% de los alumnos de control a un 12,5\% de los alumnos tratados. Esta información de carácter cuantitativo se completa con la apreciación cualitativa, llevada a cabo mediante una ficha de observación, según la cual la incorporación de las actividades de contenido histórico a la clase de física y química es un factor que contribuye a crear un buen ambiente de trabajo en el aula y aumenta la participación e interés de los alumnos (Traver, 1996). Aunque las afirmaciones de interés en los cuestionarios no significan necesariamente que haya cambiado la actitud de los alumnos, pensamos que un cierto cambio puede haberse producido al estar corrobo- 
radas las opiniones favorables por la observación de las aulas en las que se han introducido actividades de tipo histórico.

\section{CONCLUSIONES}

Con estos presupuestos, en la segunda parte del estudio nos hemos dedicado a proponer posibles soluciones al problema que tratamos. Así, hemos considerado, que si la falta de interés de los alumnos y su actitud de escasa apreciación hacia el estudio de las ciencias estaba motivada, en parte, por la visión ahistórica de la enseñanza impartida habitualmente que les mostraba una imagen sesgada de la naturaleza de la ciencia y su evolución, habría que modificar esta imagen por medio de la introducción adecuada de diversos aspectos que se pueden extraer de la historia de la ciencia y que muestren de qué manera se producen los conocimientos científicos, en qué contexto histórico y social han aparecido determinadas teorías y qué influencias han ejercido sobre el propio entorno social.

Hemos comprobado, pues, que es posible aumentar su interés hacia el estudio de la física y la química mediante un tratamiento mínimamente detenido de algunos aspectos históricos introducidos en el proceso de adquisición de los diferentes conceptos y teorías científicas, ya que así se puede mostrar una imagen de la ciencia más correcta y próxima a la realidad del trabajo de los científicos y al contexto en que éste se desarrolla y se ha desarrollado a lo largo de la historia.

\section{PERSPECTIVAS}

En primer lugar, consideramos que la historia de la ciencia puede contribuir al desarrollo y profundización del constructivismo. En efecto, éste no es una teoría cerrada y completa y, hoy por hoy, básicamente se centra en la epistemología de la ciencia y la psicología del

\section{REFERENCIAS BIBLIOGRÁFICAS}

ALIC, M. (1991). El legado de Hipatia. México: Siglo XXI editores.

CALATAYUD, M.L., HERNÁNDEZ, J., PAYA, J., SOLBES, J., TARÍN, F., y VILCHES, A. (1995, 1996, 1997 y 1998). Proyecto Galaxia: Física y Química $3^{\circ}$ ESO, Física y Química $4^{o}$ ESO, Física y Química $1^{o}$ de Bachillerato, Física $2^{o}$ de Bachillerato, Química $2^{\circ}$ de Bachillerato. Barcelona: Octaedro.

GRUP FARADAY. (1988). Física $i$ química Faraday: un enfocament conceptual, experimental $i$ històric. Barcelona: Teide. aprendizaje. En realidad se trata más bien de un programa o marco de investigación que puede y debe ser completado con la sociología e historia de la ciencia, con la sociología de la educación y un largo etcétera, que nos ayudará a comprender más la compleja realidad de la ciencia y de la educación científica.

En segundo lugar, consideramos que hay que apoyarse en la historia de las ciencias para transmitir la idea de que la ciencia es una construcción de conocimientos para resolver problemas. Pero no sólo eso. También es el conjunto de instrumentos e instituciones que permiten obtener esos resultados; y la comunidad científica que los obtiene; y no podemos dejar de lado las relaciones CTS, es decir, las relaciones de la ciencia con otros subsistemas sociales (el estado, la industria, el complejo militar, la sanidad, la educación, etc.) ni su impacto ideológico (fuente de ideas y de cambios en las creencias y opiniones de mucha gentes) (Serres et al., 1991, Ziman, 1986). Sólo así podemos dar una imagen más completa de la empresa científica, sin reducirla a sus productos, sus instrumentos y sus métodos, olvidando a las personas que trabajan en ella y sus relaciones con el contexto social y medioambiental. Desde esta visión más amplia podemos empezar a cambiar la imagen de la ciencia $y$, en consecuencia, la idea que se hacen los profesores de los objetivos de la enseñanza de las ciencias. Actualmente, para amplios sectores del profesorado de secundaria, enseñar ciencias es enseñar contenidos científicos y, en menor medida, procedimientos, es decir, básicamente una ciencia para futuros científicos, ingenieros, etc. y no una ciencia que contribuya a la formación general de todos los ciudadanos (Hodson, 1994; Solbes y Vilches, 1997). Esta preconcepción sobre las finalidades de la ciencia es uno de los problemas que plantea la enseñanza secundaria obligatoria. En una sociedad global en que la ciencia y la tecnología juegan cada vez un papel más importante, si se quiere evitar el déficit democrático que supone que la información y las decisiones sobre la ciencia estén cada vez en menos manos, es necesaria esa formación científica que permita a los ciudadanos opinar, participar, votar, etc. sobre esos temas.

HODSON, D. (1994). Seeking directions for change. The Personalisation and Politisation of Science Education. Curriculum Studies, 2(1), pp. 71-98.

IZQUIERDO, M. (1994). Cómo contribuye la historia de las ciencias en las actitudes del alumnado hacia la enseñanza de las ciencias. Aula de Innovación Educativa, 27, pp. $37-41$.

LÓPEZ PIÑERO, J.M. y NAVARRO, V. (1995). Història de la ciència al País Valencià. València: Alfons el Magnànim. 
ROMERO,F. (1998). Una pequeña reflexión sobre los problemas de investigación de didáctica de las ciencias. Enseñanza de las Ciencias, 16(1), pp. 171-174.

SCHIEBINGER, L. (1989). The Mind Has No Sex? Women in the Origins of Modern Science. Londres: Harvard University Press.

SERRES, M. et al. (1991). Historia de las ciencias. Madrid: Cátedra.

SOLBES, J. y TRAVER, M.J. (1996). La utilización de la historia de las ciencias en la enseñanza de la física y química. Enseñanza de las Ciencias, 14(1), pp. 103-112.

SOLBES, J. y VILCHES, A. (1997). STS Interactions and the teaching of Physics and Chemistry. Science Education, 81(4), pp. 377-386.
SOLSONA, N. (1997). Mujeres científicas de todos los tiempos. Madrid: Talasa ediciones.

SPECTOR, T.I. (1995). Namimg names. A brief biography of women chemist. Journal of Chemical Education, 72(5), pp. 393-395.

TRAVER, M.J. (1996). «La historia de les ciències en l'ensenyament de la física i la química». Tesi doctoral. Departament de Didàctica de les Ciències. Universitat de València.

TRUESDELL, C. (1975). Ensayos de historia de la mecánica. Madrid: Tecnos.

ZIMAN, J. (1989). Introducción al estudio de las ciencias. Barcelona: Ariel.

[Artículo recibido en mayo de 1999 y aceptado en octubre de 2000.] 


\section{Anexo}

\section{Ejemplos de actividades de historia de las ciencias}

A.1. Leer y comentar la siguiente referencia biográfica de Galileo con la ayuda de las cuestiones siguientes. (Se adjunta un texto de unas 60 líneas con la biografía.)

C1. Confeccionar una lista de los personajes que aparecen en el texto y hacer un esquema que exprese las relaciones entre ellos.

C2. Citar algún personaje histórico conocido de vuestro país o ciudad contemporáneo de Galileo.

C3. Aclarar en qué países o estados vivió Galileo, pues entonces Italia no era un sólo estado como ahora.

C4. Hacer una breve cronología con las fechas que aparecen en el texto y, paralelamente, citar hechos históricos que sean fáciles de identificar.

C5. Resumir los hechos que consideréis más significativos de la vida de Galileo.

(Texto completo y comentarios en Traver, 1996, p. 260)

A.2. Leer el siguiente cuadro y contestar las cuestiones propuestas. (Se adjunta un texto extenso sobre la ciencia española en el siglo XVI titulado «España y la revolución científica».)

C1. ¿Creéis que esta actitud de poco aprecio por la ciencia entre nosotros todavía persiste?

C2. ¿Creéis que ya hemos recuperado el retraso científico y tecnológico?

C3. Todos los procesos de recuperación en ciencias son costosos. ¿Podéis encontrar en el texto dos ejemplos de estas dificultades?

(Texto completo, Física y química $1^{\circ}$ bachillerato, en Calatayud et al., 1995 y, con comentarios, en Traver, 1996, p. 264)

A.3 Leer este texto y a partir de la información que contiene tratar de responder las cuestiones. (Se adjunta un extenso diálogo sobre la naturaleza de la luz entre Huygens y Newton imaginado por Einstein en su libro La evolución de la física.)

C1. ¿Qué significa que una onda es un transporte de energía y no de sustancia?

C2. ¿Por qué, como dice N, imaginar una onda en un espacio vacío es, en realidad, como no imaginar ninguna?

C3. Exponer lo que sepáis acerca de la velocidad de la luz y proponer algún experimento para medirla.

C4. El éter es una sustancia hipotética. ¿Qué otras sustancias hipotéticas, que se han propuesto a lo largo de la historia de la ciencia, recordáis? Enumerarlas y comentar para qué se utilizaban.

C5. Indicar cómo se podría constatar si la luz se difracta o no.

C6. Razonar qué papel tiene la longitud de onda en la difracción de la luz.

C7. Describir algún experimento, si lo conocéis, donde se pueda observar la difracción de la luz.

C8. Argumentar qué se vería en la pantalla cuando se hace pasar luz a través de un orificio si la luz estuviese constituida por partículas.

(Texto completo y comentarios en Traver, 1996, p. 293)

A.4. Leer y comentar el texto siguiente sobre con la ayuda de las cuestiones. (Se adjunta un texto extenso sobre el origen y la evolución del concepto de campo.)

C1. Hacer un esquema donde aparezcan las dos líneas de investigación que estudiaban la interacciones eléctricas, los científicos que las desarrollaron y la manera cómo se resolvió la controversia.

C2. Señalar claramente las diferencias entre la interpretación mecanicista de los fenómenos eléctricos y la nueva interpretación que introduce el concepto de campo.

C3. La visión mecanicista de los fenómenos físicos no se limitó a la interpretación de la electricidad. Comentar qué otros problemas físicos se quería explicar con esta visión, según la cual todo tiene una explicación en términos de partículas y fuerzas entre ellas.

(Texto completo en Solbes y Tarín, 1996 y, con comentarios, en Traver, 1996, p. 306)

A.5. Leer y comentar el texto siguiente con la ayuda de las cuestiones. (Se adjunta un extenso texto sobre los proyectos «Manhattan» y «Radar» y la revolución científico-tecnológica.)

C1. Valorar la contribución de la revolución científico-tecnológica a la sociedad contemporánea. 
C2. De la tecnología nuclear (bombas, misiles, etc.), Born decía que era «un triunfo de la inteligencia y un fracaso de la razón». ¿Consideráis válida esta afirmación?

(Texto completo en Solbes y Tarín, 1996 y, con comentarios, en Traver, 1996, p. 322).

A.6. Visión y comentario del vídeo «Descubriendo elementos». Cuestiones para el comentario.

C1. Citar algunas técnicas que sirvieron en diferentes momentos históricos para aislar e identificar nuevos elementos.

C2. Comentar por qué metales como el oro, la plata o el hierro ya eran conocidos en la antigüedad aunque no se consideraban elementos.

C3. Buscar información sobre los «cuatro elementos» aristotélicos y clarificar por qué actualmente no se les considera elementos.

C4. Indicar qué elementos más conocidos fueron aislados en la era neumática y con qué técnicas.

C5. Explicar por qué el análisis espectral permitió descubrir nuevos elementos.

C6. Buscar información sobre la técnica de licuefacción del aire.

(El vídeo corresponde al curso «Fundamentos de la ciencia» de la Open University, producido por la BBC. Comentarios en Traver, 1996, p. 126)

A.7. Leer el cuadro siguiente y contestar las cuestiones propuestas. (Se adjunta un extenso texto sobre la ciencia española en la Ilustración y el descubrimiento de elementos del sistema periódico.)

C1. Expresar vuestra opinión sobre las contribuciones científicas hechas en España durante la Ilustración y compararlas con las contribuciones hechas desde otros ámbitos de la cultura como el arte o la literatura.

C2. En el texto anterior hemos podido ver un colapso de la ciencia española después de una época de esplendor. ¿Conocéis algún otro caso parecido en una época anterior? ¿Cuáles podrían ser las causas?

(Texto completo, Física y química $1^{\circ}$ bachillerato, en Calatayud et al., 1995 y, con comentarios, en Traver, 1996, p. 134)

A.8. Leer, comentar y hacer un esquema con las principales ideas que contiene el siguiente texto de Lavoisier, tomado de su obra Tratado elemental de química (1789), que se suele considerar como uno de los primeros libros de química moderna.

(Se adjunta el principio del capítulo xIII, de la descomposición de los óxidos vegetales por la fermentación vinosa.)

(Texto completo y comentarios en Traver, 1996, p. 343)

A.9. Leer el texto y con la ayuda de las cuestiones comentar qué opinión os merecen el papel desarrollado por las mujeres en la creación científica a lo largo de la historia, a partir del ejemplo de Marie-Anne Lavoisier: (Se adjunta texto biográfico y foto de los esposos Lavoisier)

C1. ¿Creéis que por sus contribuciones es merecedora de tan poca consideración para no figurar la autora en la portada del libro y aparecer únicamente al pie de las figuras?

C2. ¿Conocéis otras mujeres dedicadas a la ciencia en diferentes épocas?

(Texto completo y comentarios en Traver, 1996, p. 345)

A.10. Leer este texto sobre las consecuencias sociales del proceso de Haber-Bosch y contestar las cuestiones. (Se adjunta un texto extraído de Ciencia y Sociedad de la Open University.)

C1. ¿Cuáles son las causas que hacen del nitrógeno un elemento esencial para los seres vivos? ¿Las plantas pueden tomar directamente el nitrógeno de la atmósfera? ¿Qué se entiende por nitrógeno fijo?

C2. ¿Cuáles fueron los objetivos sociales involucrados en el inicio de las aplicaciones a gran escala de las técnicas de fijación del nitrógeno?

C3. Indicar brevemente el contexto socioeconómico en que se produjo la síntesis de Haber-Bosch.

C4. ¿Qué desventajas comporta la producción actual de fertilizantes nitrogenados?

C5. ¿Qué efectos puede tener un exceso de abundancia de nitrógeno en las reservas de agua?

(Texto completo, Química COU, en Calatayud et al., 1991 y, con comentarios, en Traver, 1996, p. 350) 